\title{
THE STOKES STREAM FUNCTION IN AXIALLY SYMMETRIC POTENTIAL THEORY
}

\section{ALBERT E. HEINS $\dagger$}

\section{Introduction}

We have recently discussed in [1] a method which provides a useful transformation of the fundamental solution

$$
\frac{1}{2 \pi} \int_{0}^{\pi} \frac{\sin ^{p-1} \alpha d \alpha}{\left[(x-\gamma)^{2}+y^{2}+b^{2}-2 y b \cos \alpha\right]^{p / 2}}, \quad p>0
$$

for the equation of axially symmetric potential theory, that is

$$
\frac{\partial^{2} \phi}{\partial x^{2}}+\frac{\partial^{2} \phi}{\partial y^{2}}+\frac{p}{y} \frac{\partial \phi}{\partial y}=0, \quad p>0 .
$$

This transformation produced the Laplace representation for equation (1.2).

$$
\phi(x, y)=\frac{\Gamma((p+1) / 2)}{\sqrt{\pi} \Gamma(p / 2)} \int_{0}^{\pi} \phi(x+i y \cos \alpha, 0) \sin ^{p-1} \alpha d \alpha, \quad p>0
$$

and simultaneously indicated some of its limitations. Amongst the advantages of (1.3), however, is the fact that we may formulate boundary value problems of axially symmetric potential theory in terms of data on the axis $y=0$, without recourse, in many cases, to special functions and in terms of integral equations of the Volterra type [2].

We now turn to a discussion of the stream function for which some interesting properties were noted by A. Weinstein [3]. Here, however, we wish to show that we may give the stream function associated with $(1.1)$ in the large, in such a form that it is evident that it possesses a "barrier" from $(\gamma, b)$ to $(\gamma, \infty), b>0$. This characterization is then different from Weinstein's, since we find that the angle $\beta$ he introduced (and which he measured with respect to the line segment drawn from $(\gamma, 0)$ to $(\gamma, b)$, $b>0$ ) is limited and indeed $-\pi \leqslant \beta \leqslant \pi$.

We shall see that it is not convenient to work with (1.1) but rather with a transformed version in order to provide the form which will enable us to do this. It will then be necessary to use another transformation [1] to produce the expression we seek. Let us note that there are actually two stream functions associated with the index $p$. One of them is related to (1.1) via the Beltrami-Stokes equations and is the discontinuous one of which we have spoken. The other one arises from the correspondence principle of Weinstein [3]. This shows that the latter stream function is for $p>0$, related to the potential function of index $p+2$, rather than $p$. That is, it is a solution of

\footnotetext{
Received 3 November, 1974.
}

$\dagger$ This research was supported in part by the Air Force Office of Scientific Research, Office of Aerospace Research, USAF, under UFOSR Grant No. 71-2081. 
equation (1.2) with $p$ replaced by $p+2$. In this case, we shall find that it is the potential function of index $p$ which is discontinuous, rather than the stream function. The barrier is now drawn from $(\gamma, 0)$ to $(\gamma, b), b>0$. This situation differs in minor detail from the first one and we need only produce the fundamental solution of the equation for the stream function [equation (1.2) with $p<0$ ] in order to provide this potential function. Let us note that we have no choice of these barriers, since they are byproducts of our work.

\section{The Stream Function Associated with the Potential Function (1.1)}

We recall that the Beltrami-Stokes equations are

$$
y^{p} \frac{\partial \phi}{\partial x}=\frac{\partial \psi}{\partial y} \quad \text { and } \quad y^{p} \frac{\partial \phi}{\partial y}=-\frac{\partial \psi}{\partial x}, \quad p>0
$$

where $\phi$ is the potential function and $\psi$ is the stream function. If $\phi$ is a fundamental solution which is given by (1.1), we could, in theory, find $\psi$ from the system (2.1). Unfortunately, except for special values of $p$, we would have to investigate the properties of a double integral, in particular on the line $x=\gamma$. We can avoid this difficulty by noting [1], that (1.1) may be written in two other forms which turn out to be more useful. [We do not consider those forms expressed in terms of Bessel functions because they do not reveal, in an obvious manner, the properties of the stream function.]

Hence we write (1.1) as [1]

$$
\begin{aligned}
\phi(x, y) & =\frac{1}{2 \pi} \int_{0}^{\pi} \frac{\sin ^{p-1} \alpha d \alpha}{\left[(x-\gamma)^{2}+y^{2}+b^{2}-2 y b \cos \alpha\right]^{p / 2}} \\
& =\frac{1}{2 \pi} \int_{0}^{\pi} \frac{\sin ^{p-1} \alpha d \alpha}{\left[(x-\gamma+i y \cos \alpha)^{2}+b^{2}\right]^{p / 2}} \\
& =\frac{1}{2 \pi} \int_{0}^{\pi} \frac{\sin ^{p-1} \alpha d \alpha}{\left[(x-\gamma+i b \cos \alpha)^{2}+y^{2}\right]^{p / 2}} .
\end{aligned}
$$

These integrals are symmetric functions of $x$ and $\gamma$ as well as $y$ and $b$. This statement is obvious for (2.2a) and (2.2b) imply (2.2c) after a minor change of variables. Upon using the second Beltrami-Stokes equation we now observe that we have

$$
\psi(x, y)=\frac{-i y^{p}}{2 \pi} \int_{0}^{\pi} \frac{\cos \alpha \sin ^{p-1} \alpha d \alpha}{\left[(x-\gamma+i y \cos \alpha)^{2}+b^{2}\right]^{p / 2}}, \quad p>0
$$

(up to an arbitrary constant). Despite the presence of the factor $i$, the integral is real. $\psi(x, y)$, however, is no longer a symmetric function of the coordinates $(x, y)$ and $(\gamma, b)$. Since the denominator of (2.3) is real for $x=\gamma, 0<y<b$, it is clear that $\psi(\gamma, y)=0$. The main problem is concerned with the behaviour of $\psi(x, y)$ for $x \rightarrow \gamma$, $y>b$. Since (2.3) is not in the best possible form to discuss the properties of $\psi(x, y)$, we turn to a procedure which we recently made familiar. 
We first make the same change of variables that we made in [1]. We put $y \cos \alpha=\tau$ in (2.3) and find

$$
\begin{aligned}
\psi(x, y) & =\frac{-i}{2 \pi} \int_{-y}^{y} \frac{\tau\left(y^{2}-\tau^{2}\right)^{(p-2) / 2} d \tau}{\left[(x-\gamma+i \tau)^{2}+b^{2}\right]^{p / 2}} \\
& =\frac{1}{2 \pi} \operatorname{Im} \int_{-y}^{y} \frac{\tau\left(y^{2}-\tau^{2}\right)^{(p-2) / 2} d \tau}{\left[(x-\gamma+i \tau)^{2}+b^{2}\right]^{p / 2}} .
\end{aligned}
$$

Then we employ the bilinear transformation which produced (2.2b) from (2.2a). That is, we use the transformation $\tau=(A+B \lambda) /(C+D \lambda)$ where

$$
A=(\bar{\sigma}+y) D / 2, \quad B=-\bar{\sigma} D, \quad C=-(\bar{\sigma}+y) D /(2 y)
$$

with $\sigma=b+i(x-\gamma)$. Equation (2.4) then becomes for $x \neq \gamma, y \neq b$

$$
\psi(x, y)=\operatorname{Im} \frac{1}{2 \pi} \int_{0}^{1} \frac{2^{p-1} y^{p}(\bar{\sigma}+y-2 \bar{\sigma} \lambda)[(\lambda)(1-\lambda)]^{(p-2) / 2} d \lambda}{(2 y \lambda-\bar{\sigma}-y)\left[(y+b)^{2}+(x-\gamma)^{2}-4 y b \lambda\right]^{p / 2}} .
$$

We have, as in [1], taken $\left[(y+b)^{2}+(x-\gamma)^{2}-4 y b \lambda\right]^{p / 2}$ to be positive. Now (2.5) may be reduced to

$$
\psi(x, y)=-\frac{(x-\gamma)}{2 \pi} \int_{0}^{1} \frac{(2 y)^{p+1}[\lambda(1-\lambda)]^{p / 2} d \lambda}{\left[(2 y \lambda-y-b)^{2}+(x-\gamma)^{2}\right]\left[(y+b)^{2}+(x-\gamma)^{2}-4 y b \lambda\right]^{p / 2}} .
$$

It is from (2.5a) that we may study in detail the character of $\psi(x, y)$. Let us observe that it bears a remarkable similarity to the Poisson integral for the half-plane. To have it in a more familiar form, we put $2 y \lambda-y=\mu$. Then

$$
\psi(x, y)=\frac{-(x-\gamma)}{2 \pi} \int_{-y}^{y} \frac{\left(y^{2}-\mu^{2}\right)^{p / 2} d \mu}{\left[(\mu-b)^{2}+(x-\gamma)^{2}\right]\left[y^{2}+b^{2}+(x-\gamma)^{2}-2 b \mu\right]^{p / 2}} .
$$

Now we recall that $(x-\gamma) /\left\{\pi\left[(\mu-b)^{2}+(x-\gamma)^{2}\right]\right\}$ is the Poisson kernel for the halfplane. We could use its known properties directly to determine $\psi(x, y), x-\gamma \rightarrow 0$ from the right or the left, were it not for the fact that there is a dependence on $(x-\gamma)$ in the second term of the denominator of the integrand. On the other hand for $(x-\gamma)^{2}<y^{2}+b^{2}-2 b \mu \leqslant(y+b)^{2}$ we may write

$$
\left[y^{2}+b^{2}-2 b \mu+(x-\gamma)^{2}\right]^{-p / 2}=\left(y^{2}+b^{2}-2 b \mu\right)^{-p / 2} \sum_{n=0}^{\infty} \frac{A_{n}(x-\gamma)^{2 n}}{\left(y^{2}+b^{2}-2 b \mu\right)^{n}}
$$

where $A_{0}=1$. Now for $-y \leqslant \mu \leqslant y$, and $y>b$, we have

$$
(x-\gamma)^{2 n} /\left(y^{2}+b^{2}-2 b \mu\right)^{n} \leqslant(x-\gamma)^{2 n} /(y-b)^{2 n} \leqslant 1
$$

and the series therefore converges uniformly. Hence

$$
R(x, y, \mu)=\sum_{n=1}^{\infty} \frac{A_{n}(x-\gamma)^{2 n}}{\left(y^{2}+b^{2}-2 b \mu\right)^{n}}=O\left[(x-\gamma)^{2}\right], \quad x-\gamma \rightarrow 0, y>b .
$$

Accordingly

$$
\left[y^{2}+b^{2}-2 b \mu+(x-\gamma)^{2}\right]^{-p / 2}-\left[y^{2}+b^{2}-2 b \mu\right]^{-p / 2}=R(x, y, \mu)
$$


and therefore

$$
\begin{aligned}
\left|(x-\gamma) \int_{-y}^{y} \frac{\left(y^{2}-\mu^{2}\right)^{p / 2} R(x, y, \mu) d \mu}{\left[(x-\gamma)^{2}+(\mu-b)^{2}\right]}\right| & \leqslant|x-\gamma| \max _{|\mu|<y}|R(x, y, \mu)| \int_{-y}^{y} \frac{y^{p} d \mu}{\left[(x-\gamma)^{2}+(\gamma-b)^{2}\right]} \\
& =O(|x-\gamma|)^{2}, \quad y>b,
\end{aligned}
$$

and this approaches zero when $x \rightarrow \gamma, y>b$.

We are therefore left with the evaluation of the integral

$$
-\frac{(x-\gamma)}{2 \pi} \int_{-y}^{y} \frac{\left(y^{2}-\mu^{2}\right)^{p / 2} d \mu}{\left[(x-\gamma)^{2}+(\mu-b)^{2}\right]\left[y^{2}+b^{2}-2 b \mu\right]^{p / 2}}
$$

in the limit $x=\gamma$. Now it is known that if $x-\gamma \rightarrow 0^{+}$, the limit of $(2.6)$ is $-1 / 2$ while if $x-\gamma \rightarrow 0^{-}$, the limit is $1 / 2$. Hence

$$
\psi\left(\gamma^{+}, y\right)=-1 / 2, \quad \psi\left(\gamma^{-}, y\right)=1 / 2, \quad 0<b<y .
$$

If now $b>y>0, \psi(x, y)$ is continuous at $x=\gamma$ and indeed vanishes.

We now summarize the important properties of $\psi(x, y) . \psi(x, y)$ is a solution of the partial differential equation

$$
\frac{\partial^{2} \psi}{\partial x^{2}}+\frac{\partial^{2} \psi}{\partial y^{2}}-\frac{p}{y} \frac{\partial \psi}{\partial y}=0, \quad p>0,
$$

save in the neighbourhood of the semi-infinite straight line passing through the point $(\gamma, b)$, parallel to the $y$ axis and extending to $+\infty$. It also vanishes on the line $y=0$. We now introduce the angle $\beta$ to which we alluded in the Introduction. Weinstein has shown [3], that $\psi(x, y)=-\beta /(2 \pi)$ when $(x-\gamma)^{2}+(y-b)^{2} \rightarrow 0$, that is, when a radial limit is taken of the function $\psi$ to the point $(\gamma, b)$. Now since the form we derived for $\psi(x, y)$ is single valued and continuous in the half-plane $y>0$, save in the neighbourhood of the barrier, $\beta$ is restricted to the interval $-\pi \leqslant \beta \leqslant \pi$. The limiting values we have found for $\psi(x, y)$ on the barrier give us no other choice for $\beta$.

\section{The Stream Function with a Source and its Associated Potential Function}

Now we turn to the problem of discussing the case of a stream function with a source. In this case we recall that $\psi$ is a function which satisfies the partial differential equation

$$
\frac{\partial^{2} \psi}{\partial x^{2}}+\frac{\partial^{2} \psi}{\partial y^{2}}-\frac{p}{y} \frac{\partial \psi}{\partial y}=0, \quad p>0 .
$$

In Section 2 we have accounted for a stream function which is discontinuous on the line $x=\gamma, y>b>0$. How then can we find a stream function with a source? We accomplish this by applying Weinstein's correspondence principle, that is, we put $\psi=y^{1+p} A$ and find that

$$
\frac{\partial^{2} A}{\partial x^{2}}+\frac{\partial^{2} A}{\partial y^{2}}+\frac{p+2}{y} \frac{\partial A}{\partial y}=0 .
$$

In this case then there is a function $A(x, y)$ which satisfies equation (3.2) and which has a source at $x=\gamma, y=b$. That is, it has the form (2.2b) or (2.2c) with $p$ replaced by $p+2$. Hence there exists a function $\psi(x, y)$ which satisfies equation (3.1), which has 
the required source and is of the form

$$
\psi(x, y)=\frac{y^{1+p}}{2 \pi} \int_{0}^{\pi} \frac{\sin ^{p+1} \alpha d \alpha}{\left[(x-\gamma+i y \cos \alpha)^{2}+b^{2}\right]^{(p+2) / 2}}
$$

or

$$
=\frac{y^{1+p}}{2 \pi} \int_{0}^{\pi} \frac{\sin ^{p+1} \alpha d \alpha}{\left[(x-\gamma+i b \cos \alpha)^{2}+y^{2}\right]^{(p+2) / 2}} .
$$

Now we may use the Stokes-Beltrami equations to determine the corresponding potential. But while we used the first form (3.3a) in Section 2, we cannot use it conveniently in this case since we do not find an obviously useful form for the potential. In the present case, we use the form (3.3b) to obtain, up to an arbitrary constant, that

$$
\phi(x, y)=-\frac{1}{2 \pi} \int_{0}^{\pi} \frac{\sin ^{p+1} \alpha(x-\gamma+i b \cos \alpha) d \alpha}{\left[(x-\gamma+i b \cos \alpha)^{2}+y^{2}\right]^{(p+2) / 2}}, \quad p>0 .
$$

An integration by parts reduces (3.4a) to

$$
\phi(x, y)=-\frac{1}{2 \pi i b} \int_{0}^{\pi} \frac{\sin ^{p-1} \alpha \cos \alpha d \alpha}{\left[(x-\gamma+i b \cos \alpha)^{2}+y^{2}\right]^{p / 2}}, \quad p>0 .
$$

(3.4b) now differs from (2.3) by the presence of certain external factors and the fact that $y$ and $b$ are interchanged in the integrand. Hence the discontinuity in this integral now occurs on the line $x=\gamma, 0<y<b$, and has the same general character that we found for $\psi(x, y)$ in Section 2. Now, however, we measure the angle $\beta$ with respect to the line $x=\gamma, y>b$, so that the right side of the segment $x=\gamma, 0<y<b$ is $\beta=-\pi$ and the left side is $\beta=\pi$. We have then that $\phi(x, y)=0$ for $x=\gamma, y>b$. For $x-\gamma \rightarrow 0^{+}, 0<y<b$, we get that

$$
\phi(x, y)=b^{-p-1} / 2
$$

and for $x-\gamma \rightarrow 0^{-}, 0<y<b$, we have

$$
\phi(x, y)=-b^{-p-1} / 2 .
$$

Weinstein has provided an interpretation for such integrals as (2.3) and (3.4b) in terms of special forms of the Weber-Schafheitlin integrals.

\section{References}

1. A. E. Heins, "The fundamental solution in axially symmetric potential theory ", Proc. London Math. Soc., to appear.

2. ___ "Axially symmetric boundary value problems ", Bull. Amer. Math. Soc., 71 (1965), 787808.

3. A. Weinstein, "Discontinuous integrals and generalized potential theory", Trans. Amer. Math. Soc., 63 (1948), 342-354.

Department of Mathematics, University of Michigan,

Ann Arbor, MI 48104. 